\title{
Manejo dental a ocelote; tratamiento de conductos radiculares y rehabilitación: reporte de caso
}

\section{Dental management to ocelot; root canal treatment and rehabilitation: case report}

\begin{abstract}
Iván H. Medina-Ordaz ${ }^{a}$, María F. Hernández-Salgado ${ }^{b}$,Erick A. Acoltzin-Chavez ${ }^{c}$
Abstract:

The oral cavity represents the entrance to the digestive system and any disfunction, disease or abnormality has the power to cause an unfavourable impact on the health of the animals. In order to contribute to the conservation of the species, dental care in wildlife is essential to optimize an adequate nutritional status. Clinical case of veterinary dentistry is presented in ocelot, species of felines that are in protection category, procedures such as root canal treatment, teeth rehabilitation were performed in order to restore function and avoid oral problems, guaranteeing animal welfare.
\end{abstract}

Keywords:

Dental care, ocelot, conservation, animal welfare, root canal treatment.

\begin{abstract}
Resumen:
La cavidad oral representa la entrada al sistema digestivo y cualquier disfunción, enfermedad o anormalidad tiene la facultad de ocasionar un impacto desfavorable sobre la salud de los animales. Con la finalidad de coadyuvar a la conservación de las especies, la atención dental en fauna silvestre es indispensable para optimizar un estado nutricional adecuado. Se presenta caso clínico de odontología veterinaria en ocelote, especie de felinos que se encuentran en categoría de protección, donde se realizaron procedimientos como tratamiento de conductos radiculares y rehabilitación de dientes con la finalidad de devolver función y evitar problemas bucodentales mayores, garantizando el bienestar animal.
\end{abstract}

\section{Palabras Clave:}

Atención dental, ocelote, conservación, bienestar animal, tratamiento de conductos.

\section{Introducción}

En todo el mundo la odontología veterinaria ha experimentado una rápida evolución y una creciente sofisticación de los productos médicos en busca de condiciones cada vez mejores para mantener la salud de los animales domésticos y silvestres [1]. La odontología veterinaria es una especialidad biomédica que se encarga de diagnosticar y tratar las patologías presentes en el aparato estomatognático de los animales. Estas patologías constituyen la tercera causa más común de afecciones médicas animales en Norteamérica [2]. La atención odontológica ha sido tradicionalmente enfocada a prevenir y resolver las afecciones orales del ser humano, según sus necesidades, tanto curativas como preventivas. En el contexto actual, donde cada vez más hay fuertes relaciones entre la vida animal y el ser humano, surge la necesidad de velar y extender la atención odontológica a especies animales tanto menores como mayores, así como a

${ }^{a}$ Cirujano dentista de practica privada egresado de la Universidad Autónoma del Estado de Hidalgo, https://orcid.org/0000-0002-93902390, Email: me230459@uaeh.edu.mx

${ }^{\mathrm{b}}$ Cirujano dentista de práctica privada egresada de la Universidad Autónoma del Estado de Hidalgo, https://orcid.org/0000-0002-4044-8040, Email: he248137@uaeh.edu.mx

${ }^{\mathrm{c}}$ Biólogo de práctica privada egresado de la Universidad Autónoma del Estado de Hidalgo, https://orcid.org/0000-0003-0009-4505, Email: ac301770@uaeh.edu.mx

*Autor de Correspondencia: me230459@uaeh.edu.mx 
animales domésticos y silvestres, que se encuentran en su hábitat o en cautiverio [3].

El propósito de esta publicación es describir y compartir las patologías y tratamientos del aparato estomatognático en una ocelote, especie de felinos silvestres, quien fue rescatada y rehabilitada por el "Centro de rescate, rehabilitación y reubicación Invictus", perteneciente a la "A.C. Fundación Invictus", hospital de alto nivel para fauna silvestre, específicamente para víctimas de incidencia humana, ya sea nacidos en cautiverio o de vida libre. El nosocomio, único en América Latina se encuentra en la de la ciudad de Pachuca de Soto, México.

La Fundación Invictus es un hospital con quirófano propio para rehabilitar a los animales que en su mayoría han logrado rescatar desnutridos, con lesiones o fracturas y sumamente maltratados. Posteriormente a su rehabilitación son reubicados a un santuario ubicado en Colorado, Estados Unidos y los pacientes que, a criterio de especialistas, sean viables a estar en vida libre, son liberados en su hábitat, todo esto con la finalidad de contribuir a la conservación de especies.

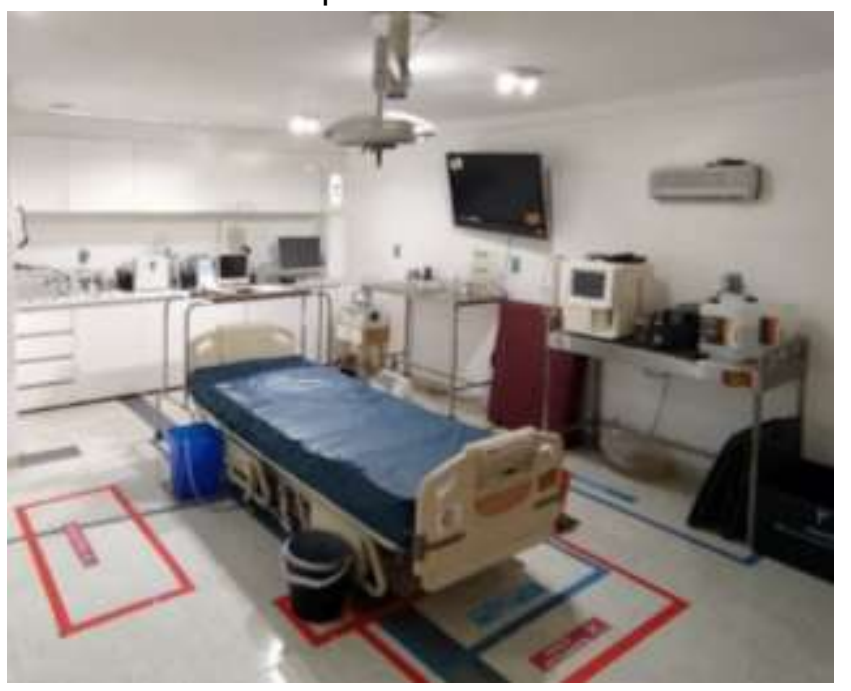

Figura 1. Quirófano de "Fundación Invictus"

México es un ejemplo claro del deterioro que han sufrido las poblaciones de grandes depredadores, cuatro de las especies de felinos silvestres que habitan sus zonas tropicales se encuentran en alguna categoría de riesgo. El jaguar (Panthera onca), tigrillo (Leopardus wiedii) y jaguarundi (Pumayaguaroundi) están en el apéndice CITES I (Convención sobre el comercio internacional de especies amenazadas de fauna y flora silvestres) y el ocelote (Leopardus pardalis) en el apéndice II de CITES [4]. En el apéndice I se incluyen las especies sobre las que se cierne el mayor grado de peligro entre las especies de fauna incluida en los apéndices de CITES mientras que en el apéndice II figuran especies que no están necesariamente amenazadas de extinción pero que podrían estarlo a menos que se controle estrictamente su comercio [5].

Éstas cuatro especies también mantienen en la legislación mexicana el estatus de especies en peligro de extinción o amenazadas, y su cacería está prohibida [4]. Así mismo, el tigre de bengala (panthera tigris) figura en peligro según el criterio A2abcd de la "International Union for Conservation of Nature's" IUNC [6].

El aparato estomatognático de animales en cautiverio es tratado comúnmente de manera preventiva con la finalidad de mejorar su calidad de vida y evitar problemas futuros. La odontología se ha desarrollado en la ejecución de técnicas y procedimientos de perfecta aplicabilidad a la asistencia de pequeños y grandes animales.

Está claro que muchos procedimientos y medicamentos modernos, no existirían sin la ayuda de la experimentación con animales. Curiosamente, como la odontología humana ha crecido directamente de esta ayuda, también la odontología veterinaria ha crecido indirectamente. Esto se vuelve cada vez más importante a medida que nos esforzamos por mejorar nuestra comprensión entre los animales y el hombre [7].

\section{Materiales y metodos}

A continuación se describirá un caso clínico de procedimientos odontológicos complejos en un paciente felino (Ocelote).

Caso clínico: Consiste de una hembra ocelote (Leopardus pardalis) de quien se desconoce su edad pues es un ejemplar que fue rescatado tras ser víctima de violencia humana, donde PROFEPA Delegación Oaxaca recibió el reporte y en coordinación con Fundación Invictus fue posible el rescate.

Por instinto natural de sobrevivencia la ocelote buscaba alimento, fue así que personas de la zona refieren haberla visto "atacar" a gallinas, 
posteriormente fue atrapada, amarrada y maltratada (Figura 2). Aparte de las lesiones observadas en el hocico, a nivel ocular presentaba una lesión importante (Figura 3).

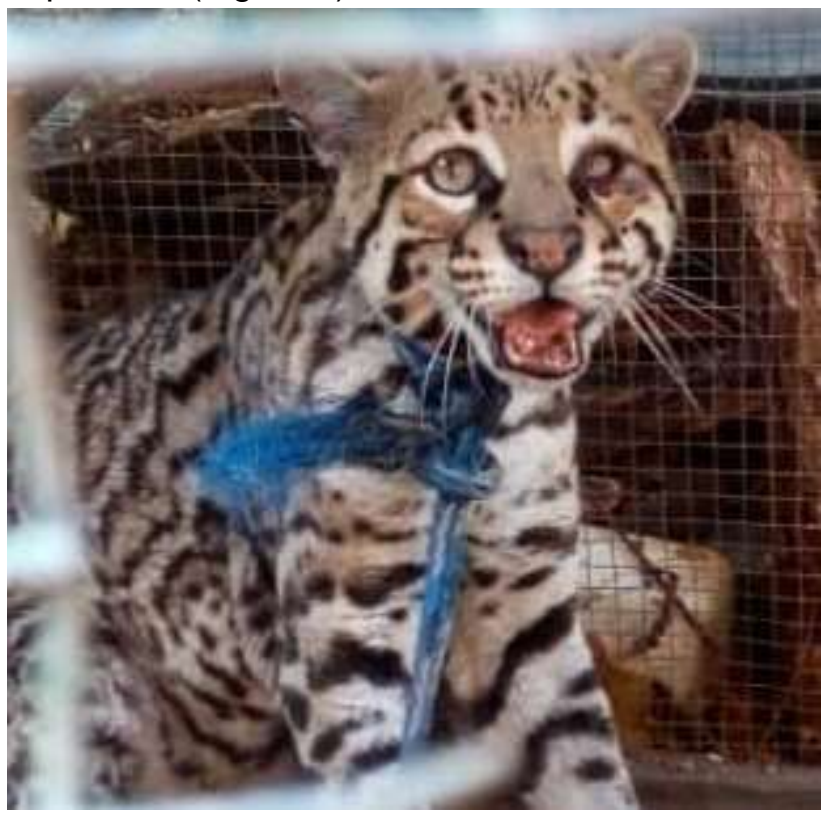

Figura 2. Ocelote hembra (Leopardus pardalis) previo a la intervención.

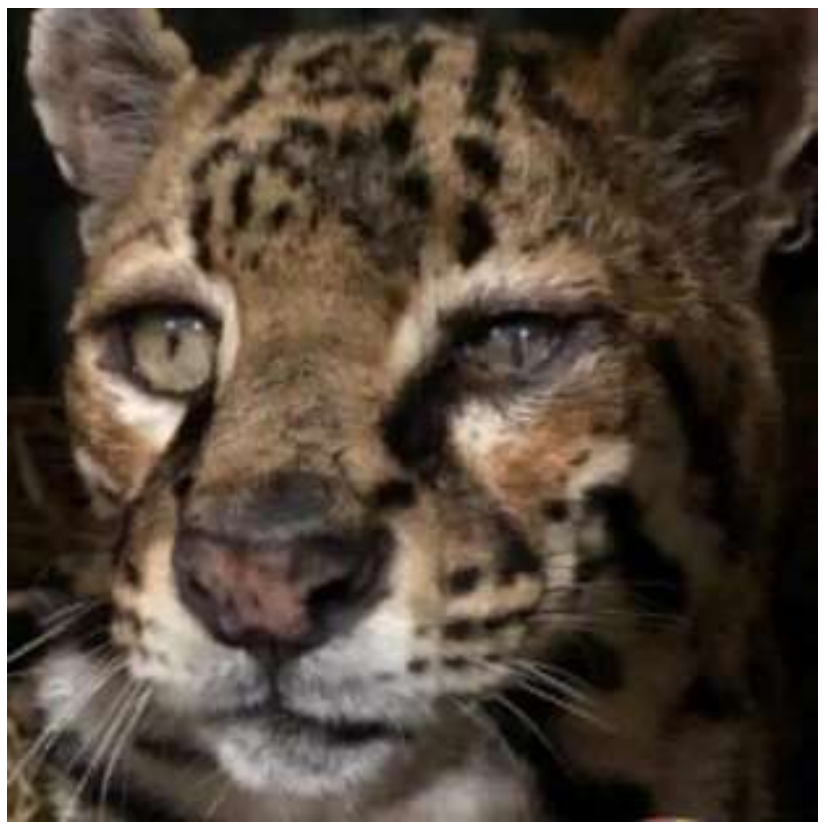

Figura 3. Lesión a nivel ocular.

Tras el rescate, las características físicas que mostraba dejaban en evidencia que no recibía alimentación, pues se enfrentaba a un estado crítico de desnutrición. Al observar la severa situación en la que se encontraba se tomó la decisión de someterla a un protocolo anestésico utilizando 0.6
$\mathrm{mL}$ de Dexmedetomidina $(0.03 \mathrm{mg} / \mathrm{kg} \quad \mathrm{PV}$ Dexdomitor ${ }^{\circledR}$ ORION PHARMA) $+0.2 \mathrm{ml}$ de ketamina (2 mg/kg PV Anesket $®$ PISA), al no ser intubada se contaba con un tiempo de trabajo limitado para tratar todas las necesidades terapéuticas que requería la ocelote (Figura 4), pues la finalidad era atender la mayor cantidad de problemas que presentaba en ese momento.

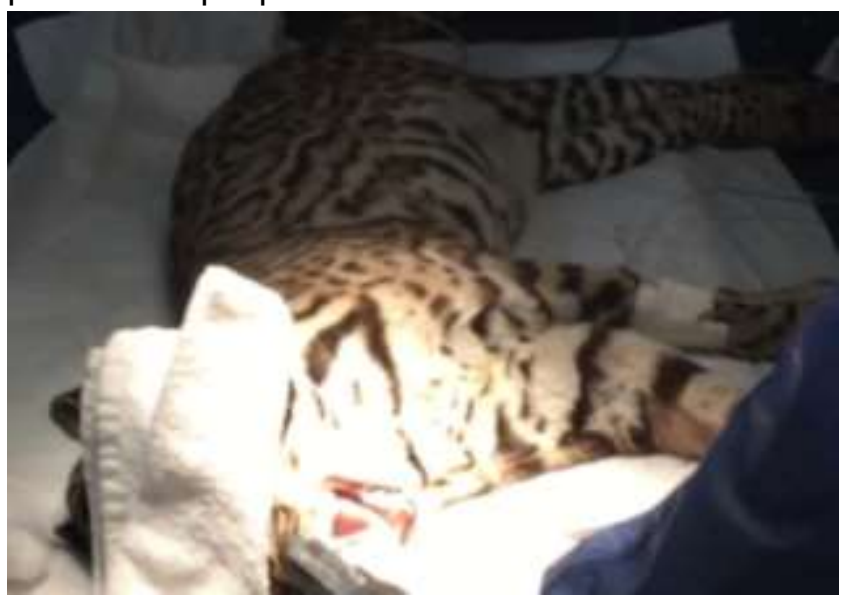

Figura 4. Inicio de protocolo de manejo a ocelote.

Debido al traumatismo que presentaba en el cráneo (Figura 5) se le realizó estudio radiográfico donde se descartó cualquier tipo de fractura ósea (figura $6)$.

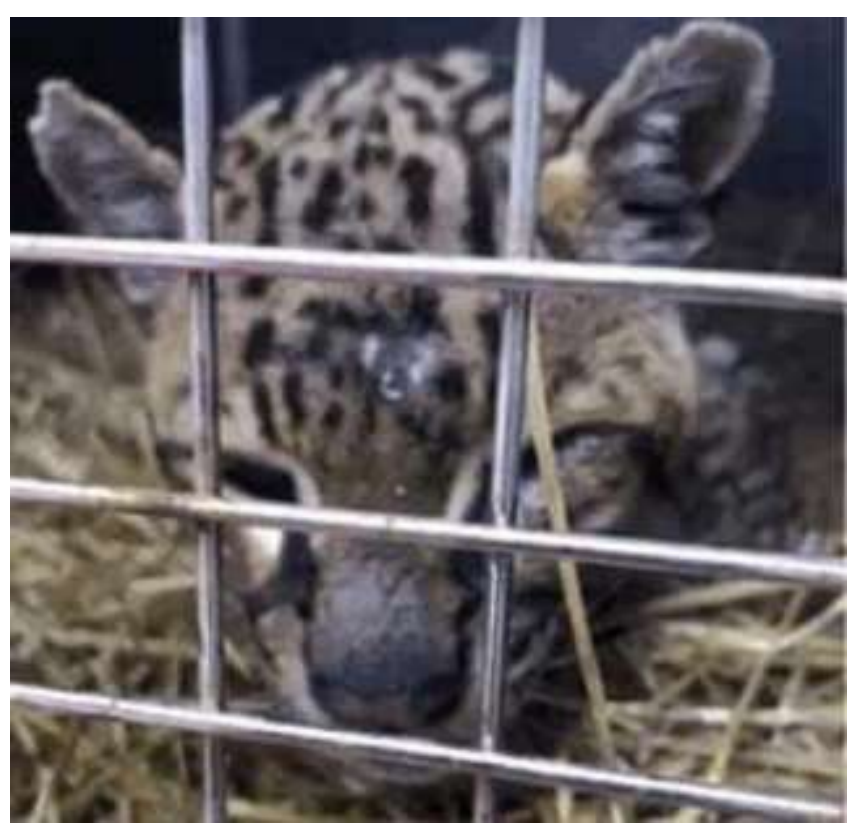

Figura 5. Traumatismo en zona frontal del cráneo. 


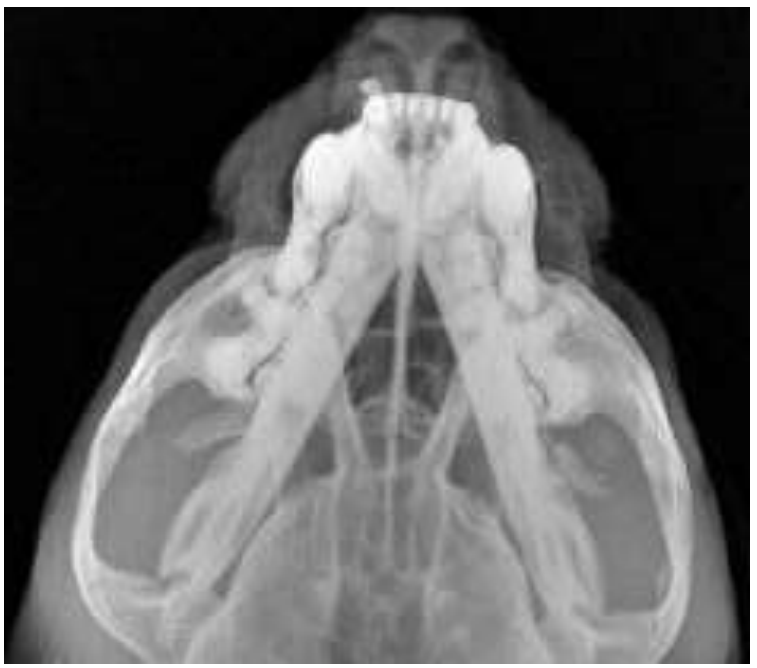

Figura 6. Radiografía de cráneo.

A nivel estomatológico, presentaba placa dentobacteriana, gingivitis generalizada, caries, edentulismo parcial en zona anteroinferior $y$ fracturas complicadas en los cuatro caninos (diente 104, 204, 304, 404). Al presentar muchas complicaciones en el aparato estomatognático y contar con un tiempo limitado de trabajo, se decidió llevar a cabo tratamientos prioritarios para mantener la salud bucodental, es por esto que se realizó una profilaxis dental con ayuda de instrumental ultrasónico (Cavitron DTE® D5) e instrumental rotatorio de baja velocidad (Turbina W\&H®).

Así mismo se realizaron tratamientos de conductos en los cuatro órganos dentales fracturados, la realización de dichos tratamientos fueron realizados con ayuda de instrumental rotatorio de alta velocidad $(\mathrm{W} \& \mathrm{H} \AA)$ con fresa en forma de bola \#2 de carburo (MDT®) como un agente de corte para una correcta apertura cameral, enseguida, para limpiar los conductos se utilizaron limas (K-FILES, MANI) de $31 \mathrm{~mm}$ primera y segunda serie, utilizando hipoclorito de sodio al $2.5 \%$ (Hertz $\left.{ }^{\circledR}\right)$ y gluconato de clorhexidina al $2 \%$ (Consepsis ${ }^{\circledR}$ ) como agentes de irrigación; el hipoclorito de sodio fue activado con frecuencia ultrasónica (Ultrasonido DTE y puntas de activación) finalmente el procedimiento de obturación, por su sencillez y seguridad, fue efectuado con técnica lateral (Figura 7) utilizando como cono maestro una punta de gutapercha estandarizada tamaño mediano (Metabiomed®) embebido en material de sellado con el que se buscó cubrir las paredes laterales previo a llenar el conducto en su totalidad, posteriormente con apoyo de un espaciador se comprimió la gutapercha contra las paredes del conducto, generando espacio para la colocación de cono accesorios.

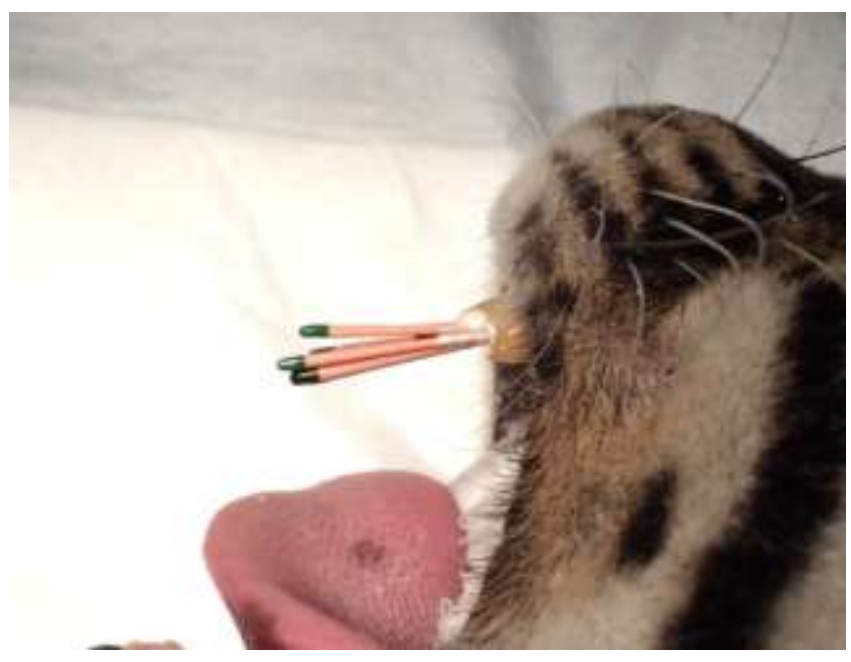

Figura 7. Obturación de conducto con tecnica lateral.

Al haber trascurrido 150 minutos desde que se inició el protocolo anestésico, el ocelote empezaba a mostrar reflejos lo cual impedía una manipulación idónea de los instrumentos dentro de boca, por lo que se le administro $0.2 \mathrm{ml}$ de Ketamina $(2 \mathrm{mg} / \mathrm{kg}$ PV Anesket @ PISA) para generar mayor tiempo de trabajo.

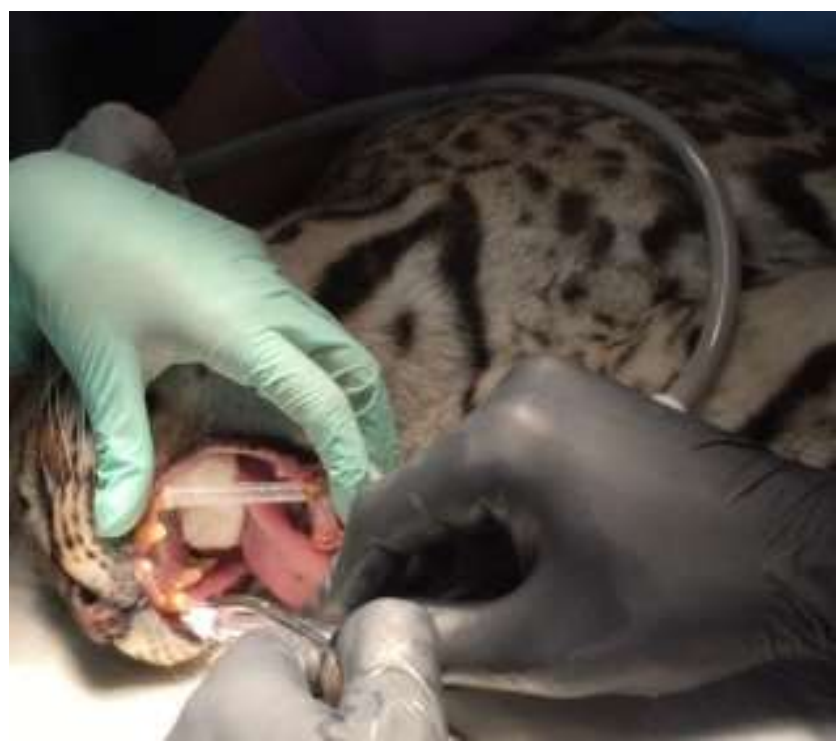

Figura 8. Ejecución de protocolo adhesivo.

Finalmente para devolver función se reconstruyeron los cuatro caninos fracturados con ayuda de un protocolo adhesivo de quinta generación (Figura 9), dicho protocolo se realizó con el sistema de TeEconom Bond (Ivoclar Vivadent ${ }^{\circledR}$ ) y resina 
compuesta Te-Econom Plus (Ivoclar Vivadent ${ }^{\circledR}$ ) pues los órganos dentales que estaban comprometidos son piezas que desarrollan un papel esencial en la alimentación, pues la función de las mismas es desgarrar el alimento.

\section{Resultados}

Finalizados los tratamientos de conductos y la rehabilitación de los mismos órganos dentales (Figura 9) se llevó a cabo un control y seguimiento conductual estricto; se observó que mejoró considerablemente su alimentación, pues al tener caninos rehabilitados podía desgarrar su alimento sin ningún problema, por ende mejoró considerablemente su condición nutricional y su estado de salud general teniendo una mejor calidad de vida.

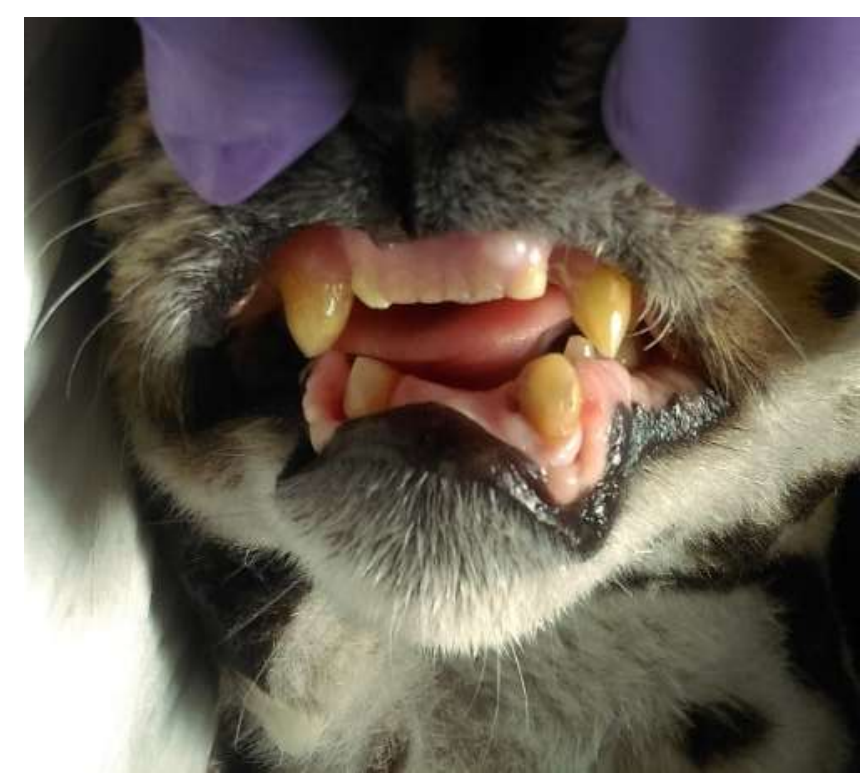

Figura 9. Resultado final de la rehabilitación dental realizado con material polimerizable (resina).

\section{Discusión}

Existe una gran diferencia entre las condiciones de un animal en su hábitat natural que las de un animal en cautiverio pues afecciones que sufra cualquier especie en su hábitat natural deberá adaptarse a diferencia de un animal que está en cautiverio, pues existe la posibilidad de ser sometido a protocolos de atención para mejorar su calidad de vida.
Hemos evidenciado la necesidad de brindar atención a animales realizando un correcto diagnóstico de las patologías que afectan su sistema estomatognático, basado en un examen minucioso de la cavidad oral.

A medida que la sociedad valora cada vez más a los animales de nuestros ecosistemas, nos damos cuenta de que estas mejoras benefician directamente a la vida silvestre. No solo es prudente monitorear los avances actuales en este campo, sino que también vale la pena explorar los comienzos de la odontología veterinaria [7].

Problemas de menor magnitud ocasionan molestia y conllevan a un menor aprovechamiento nutricional, pérdida de peso y, consecuentemente, disminución de la calidad de vida; mientras que padecimientos más severos, pueden generar a alteraciones sistémicas severas e incluso la muerte. Investigaciones recientes vienen relacionando la salud oral con la sistémica, ya que, además de la incomodidad causada en el animal, existe fuerte evidencia circunstancial indicativa que demuestra que un foco de infección en la boca puede causar la alteración y daño en órganos distantes. Se ha determinado que en la especie humana el $40 \%$ de los casos de endocarditis bacterianas provienen de infecciones primarias de la cavidad oral [9], tomando este indicador como referencia podemos determinar que la odontología veterinaria no solo constituye una serie de medidas profilácticas como la eliminación del tártaro, sino que incluye una serie de procedimientos complejos, tales como el tratamiento de conductos, extracciones, la rehabilitación de órganos dentales, entre otros, con la finalidad de evitar situaciones patológicas que comprometan la vida de los animales.

\section{Conclusiones}

Como sociedad debemos darle una mayor importancia a la protección de nuestra fauna, ayudar a su bienestar proporcionándoles una vida más saludable y larga en este caso a través del cuidado de la cavidad oral. Los animales al igual que los humanos, deben recibir atención odontológica, no atender sus afecciones bucales podría traer varios riesgos tanto en su alimentación como en su vida. Así mismo el gremio profesional 
veterinario debe seguir actualizando sus conocimientos para poder ejecutar tratamientos dentales garantizando el bienestar animal.

Por esta razón a través de esta publicación, con la ayuda de la "Fundación Invictus", su equipo interdisciplinario y mediante la presentación de dos casos clínicos de odontología veterinaria, buscamos otorgar un panorama más amplio y gráfico de una rama poco común, pero que hoy en día tiene un papel muy importante en el ecosistema. Inspirar a docentes, estudiantes y profesionales del gremio a que muestren interés por este campo de atención clínica odontológica en fauna silvestre, principalmente propiciar nuevos proyectos de investigación en atención preventiva, curativa y restaurativa con trabajo de campo tanto para especies ya estudiadas como para otras nuevas, darles continuidad a los tratamientos ya efectuados, con el fin de darles una mejor calidad de vida; este sería un paso más al futuro de esta rama de la odontología que sin duda la gran fauna de nuestro planeta lo agradecería.

\section{Agradecimientos}

Los autores agradecen a "A.C. Fundación Invictus" y a su presidenta la activista conservacionista Erika Ortigoza Vázquez por la confianza depositada y por abrir las puertas de su quirófano, felicitándola por su admirable iniciativa.

\section{Conflicto de intereses}

Los autores declaran no tener ningún conflicto de interés.

\section{Referencias}

[1] Elza M Galvão Ciffoni, Pachaly JR. Consideraciones Históricas Y Legales Sobre La Odontología Veterinária En Brasil. Vol. 4, Arq. Ciên. vet. zool. UNIPAR. 2001. p. 49-54.

[2] Zamira A. Calderón S, María V. Crespo M, Glevi Y. Montilla G, Ingeborg Paris M. IPRG. Odontología veterinaria: Revisión de la literatura. Rev Venez Investig Odontológica [Internet]. 2014;2(1):46-59. Available from: http://erevistas.saber.ula.ve/index.php/rvio/article/view/4842

[3] Marco M. Bermúdes, Randall A. Porras RPS. Odontología veterinaria en especies silvestres del Parque Zoológico Nacional Simón bolívar y Universidad Latina de Costa Rica. Odontol Vital. 2018;1(August):55-63.

[4] Ávila-Nájera DM, Chávez C, Lazcano-Barrero MA, PérezElizalde S, Alcántara-Carbajal JL. Estimación poblacional y conservación de felinos (Carnivora: Felidae) en el norte de Quintana Roo, México. Rev Biol Trop. 2015;63(3):799.

[5] CITES. Apendices I, II and III. Conv Int Trade Endanger Species Wild Flora Fauna. 2019;41(22):1-69.
[6] Uicn UMPLN. Categorías y Criterios de la Lista Roja de la UICN. 2001. 38 p.

[7] Easley K. Veterinary dentistry: its origin and recent history. J Hist Dent. 1999;47(2):83-5.

[8] Lina Marcela Rodriguez Jimenez A del PUD. Odontología en equinos: generalidades e importancia en medicina veterinaria. Rev Med Vet. 2011;22(M):69-83.

[9] Grandez R, Porras C. Frecuencia de alteraciones dentales y periodontales en perros atendidos en la clínica veterinaria de la Universidad Peruana Cayetano Heredia durante mayo - octubre 2006. Salud y Tecnol Vet [Internet]. 2015;1(1):19. Available from:

https://www.researchgate.net/publication/275833554\%0AFrecue ncia 